\title{
Qualidade do sono em jogadores de basquetebol em cadeira de rodas
}

\author{
Sleep quality in wheelchairr basketball players \\ Calidad del sueño en jugadores de baloncesto en silla de ruedas
}

Recebido: 22/11/2021 | Revisado: 02/12/2021 | Aceito: 06/12/2021 | Publicado: 19/12/2021

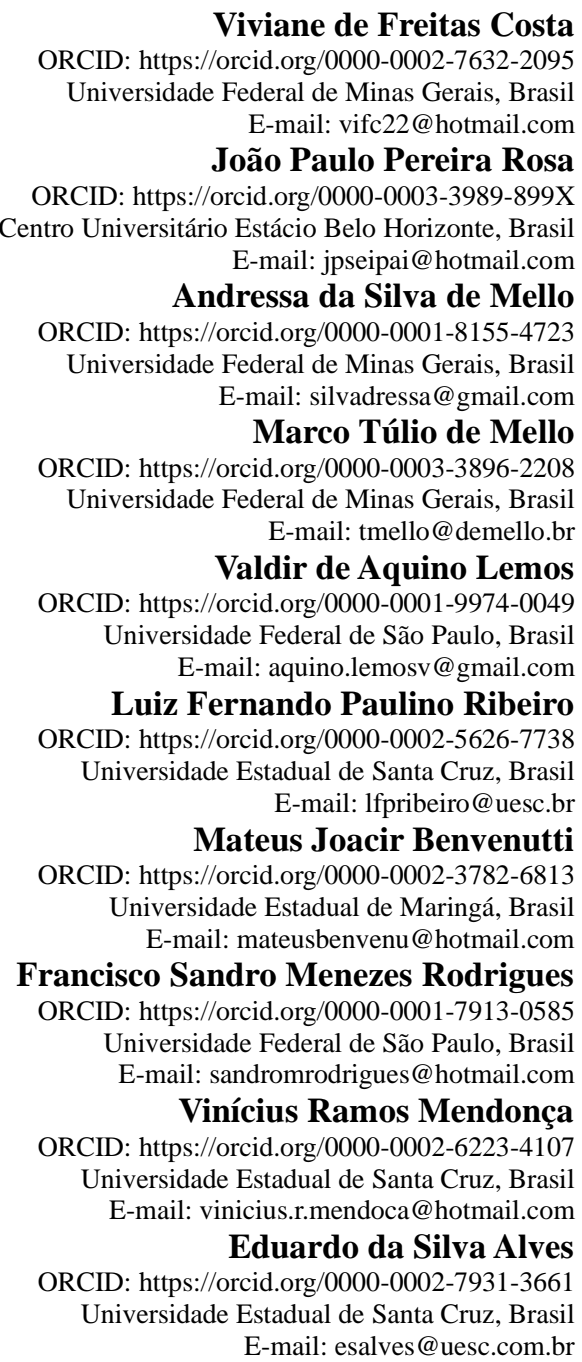

\begin{abstract}
Resumo
Introdução: A privação ou restrição do sono, reflete na qualidade de vida das pessoas e no aumento da sonolência diurna. Por outro lado, o exercicio físico contribui na melhora da qualidade do sono. Um sono de baixa qualidade é mais prevalente em pessoas com lesão medular (LM) do que na população sem LM. Objetivo: Avaliar a qualidade do sono e a sonolência em Jogadores de basquetebol em cadeira de rodas (JBCR). Métodos: A amostra foi constituída por 11 voluntários do sexo masculino, atletas de basquete em cadeira de rodas, com LM completa ou incompleta de torácica 5 (T5) a lombar 1 (L1). Os voluntários responderam ao questionário de Qualidade do Sono de Pittsburgh (PSQI) e para avaliar a sonolência diurna foi utilizada a versão traduzida da Escala de Sonolência de Epworth. Foi utilizada uma estatística descritiva para apresentação dos dados. Resultados: 8 atletas (72\%), tem a qualidade de sono ruim atingindo pontuações acima de 5 no PSQI. Com relação ao tempo total de sono, os resultados encontrados mostram uma duração do sono ou tempo total de sono (TTS) de 7,5 horas. A eficiência do sono foi de $84 \%$ e 7 atletas (63\%) apresentaram latência superior a 15 minutos. Conclusão: Os JBCR apresentam uma qualidade de sono ruim, como demonstrado pelo PSQI. Entretanto, nós acreditamos que a prática regular do exercício físico pode ter atenuado os efeitos deletérios da LM sobre o sono.
\end{abstract}

Palavras-chave: Sono; Treinamento físico; Lesões da medula espinhal. 


\begin{abstract}
Introduction: Sleep deprivation or restriction reflects on people's quality of life and increased daytime sleepiness. On the other hand, exercise contributes to improving sleep quality. Poor quality sleep is more prevalent in people with spinal cord injury (SCI) than in the population without SCI. Objective: To evaluate sleep quality and sleepiness in Wheelchair Basketball Players (JBCR). Methods: The sample consisted of 11 male volunteers, basketball athletes in a wheelchair, with complete or incomplete SCI from thoracic 5 (T5) to lumbar 1 (L1). The volunteers answered the Pittsburgh Sleep Quality Questionnaire (PSQI) and the translated version of the Epworth Sleepiness Scale was used to assess daytime sleepiness. Descriptive statistics were used to present the data. Results: 8 athletes (72\%) have poor sleep quality reaching scores above 5 on the PSQI. Regarding the total sleep time, the results found show a sleep duration or total sleep time (TTS) of 7.5 hours. Sleep efficiency was $84 \%$ and 7 athletes (63\%) had latency longer than 15 minutes. Conclusion: The JBCR have poor sleep quality, as demonstrated by the PSQI. However, we believe that regular physical exercise may have attenuated the deleterious effects of ML on sleep.
\end{abstract}

Keywords: Sleep; Physical training; Spinal cord injuries.

\title{
Resumen
}

Introducción: La privación o restricción del sueño se refleja en la calidad de vida de las personas y en el aumento de la somnolencia diurna. Por otro lado, el ejercicio contribuye a mejorar la calidad del sueño. El sueño de mala calidad es más frecuente en personas con lesión de la médula espinal (LME) que en la población sin LME. Objetivo: evaluar la calidad del sueño y la somnolencia en jugadores de baloncesto en silla de ruedas (JBCR). Métodos: La muestra estuvo constituida por 11 voluntarios varones, deportistas de baloncesto en silla de ruedas, con LME completa o incompleta desde torácica 5 (T5) a lumbar 1 (L1). Los voluntarios respondieron el Cuestionario de Calidad del Sueño de Pittsburgh (PSQI) y se utilizó la versión traducida de la Escala de Somnolencia de Epworth para evaluar la somnolencia diurna. Se utilizaron estadísticas descriptivas para presentar los datos. Resultados: 8 deportistas (72\%) tienen mala calidad del sueño alcanzando puntuaciones superiores a 5 en el PSQI. En cuanto al tiempo total de sueño, los resultados encontrados muestran una duración del sueño o tiempo total de sueño (TTS) de 7,5 horas. La eficiencia del sueño fue del $84 \%$ y 7 atletas $(63 \%)$ tuvieron una latencia de más de 15 minutos. Conclusión: los JBCR tienen una mala calidad del sueño, como lo demuestra el PSQI. Sin embargo, creemos que el ejercicio físico regular puede haber atenuado los efectos deletéreos del LME sobre el sueño.

Palabras clave: Sueño; Entrenamiento físico; Lesiones de la médula espinal.

\section{Introdução}

O sono é uma função biológica e fundamental na consolidação da memória, na termorregulação, na conservação e restauração da energia do organismo como um todo (Martins et al., 2001; Nicolau et al., 2000). Por outro lado, um sono de má qualidade pode levar a prejuízos cognitivos, comportamentais e físicos, diminuindo a qualidade de vida (Antunes et al., 2008). Somado a isso, uma baixa qualidade do sono pode estar associada com a sonolência diurna excessiva, o que resulta em fadiga além da possibilidade de aumentar o risco de acidentes de trabalho e trazer prejuízos à saúde tais como obesidade, alterações psicológicas além do aumento de risco cardiovascular (Antza et al., 2021; Müller \& Guimarães, 2007; Tobaldini et al., 2017).

Um sono de baixa qualidade é mais frequente em indivíduos com lesão medular (LM) do que na população sem deficiência, o que poderia contribuir para uma baixa qualidade de vida nesta população (Alves et al., 2005). Indivíduos com LM relatam significativamente maiores distúrbios do sono, com menos horas de sono por noite, pior qualidade de sono e sonolência diurna aumentada (Giannoccaro et al., 2013; Sankari et al., 2019). Nesse sentindo, estratégias para otimizar a qualidade do sono nesses indivíduos com LM seria de extrema importância.

Estudos prévios do nosso grupo, têm mostrado que o exercício físico pode ser utilizado como terapia não farmacológica para o tratamento dos distúrbios do sono, melhorando a sua qualidade na população em geral (Mello \& Tufik, 2004). Apesar da American Sleep Disorders Association reconhecer a importância do exercício físico como uma intervenção não farmacológica para a melhoria do padrão de sono (Chennaoui et al., 2015; Martins et al., 2001), ainda são poucos os profissionais de saúde que recomendam e prescrevem o exercício físico com essa finalidade, em especial para indivíduos com LM.

Frente a este cenário, acredita-se ser de extrema relevância a avaliação da qualidade do sono em atletas com LM, pois nossa hipótese é que a prática regular de exercício físico pode atenuar os efeitos deletérios da LM sobre a qualidade do sono. 
Sendo assim, o objetivo deste estudo é o de avaliar a qualidade do sono e a sonolência em jogadores de basquetebol em cadeira de rodas $(\mathrm{JBCR})$.

\section{Metodologia}

Todos os procedimentos desse estudo foram aprovados pelo comitê de Ética em pesquisa (0294/11), da Universidade Federal de São Paulo e estão de acordo com as normas brasileiras para pesquisas em seres humanos (Resolução $n^{\circ} 466 / 2012$ ). Todos os voluntários assinaram um termo de livre consentimento para a participação no estudo. A amostra consistiu em 11 voluntários do sexo masculino com lesão medular completa ou incompleta de torácica 5 (T5) a lombar 1 (L1). Todos os voluntários eram jogadores de basquete em cadeira de rodas, e estavam participando da pré-temporada.

Para avaliar a qualidade do sono da amostra, foi utilizada a versão traduzida o Índice de Qualidade do Sono de Pittsburgh (PSQI) (Bertolazi et al., 2011). As questões do PSQI formam sete componentes, que são analisados a partir de instruções para pontuação de cada um desses componentes, variando de zero a três pontos. A soma da pontuação máxima desse instrumento é de 21 pontos, sendo que os escores superior a cinco pontos indicam uma qualidade ruim no padrão de sono. Além disso, os atletas reportaram o tempo total de sono, latência para o sono e também foi calculada a eficiência do sono dos mesmos. Para avaliação da sonolência diurna foi utilizada a versão traduzida da Escala de Sonolência de Epworth (Bertolazi et al., 2009). Resultados entre 0 e 10 pontos indicam ausência de sonolência; entre 10 e 16 pontos, sonolência leve; entre 16 e 20 pontos, sonolência moderada e entre 20 e 24 pontos, sonolência severa.

Devido ao pequeno tamanho amostral dos dados, foi realizada uma estatística descritiva por meio da mediana, intervalo interquartis, mínimo e máximo (Callegari-Jacques, 2003).

\section{Resultados}

A Tabela 1 mostra as características gerais da amostra bem como os dados extraídos do questionário PSQI e do Epworth.

Tabela 1: Características do sono do grupo de atletas de basquetebol com lesão medular.

\begin{tabular}{|c|c|c|c|c|c|c|}
\hline & Mediana & Mínimo & Máximo & Quartil 1 & Quartil 3 & $\begin{array}{c}\text { Valores de } \\
\text { ref* }\end{array}$ \\
\hline Peso (kg) & 60.6 & 50.1 & 79.2 & 55.2 & 66.2 & - \\
\hline Altura (cm) & 168 & 158,5 & 183 & 164,5 & 175,5 & - \\
\hline Idade (anos) & 30 & 18 & 39 & 22,5 & 33 & - \\
\hline IQS & 6 & 1 & 13 & 4,5 & 8 & $<5$ \\
\hline TTS (horas) & 7,5 & 5 & 10 & 6 & 8 & $7-9$ \\
\hline Eficiência do sono (\%) & 84 & 40 & 100 & 76 & 93 & $>85$ \\
\hline Latência do sono (min) & 20 & 2 & 60 & 7,5 & 30 & $<15$ \\
\hline Sonolência & 8 & 3 & 16 & 5 & 9 & $<10$ \\
\hline
\end{tabular}

Kg= Quilograma; Cm= Centímetro; IQS= Índice de Qualidade de Sono; TTS= Tempo Total de Sono; < = menor; > = maior

* Valores de referência baseado no índice de qualidade de sono de Pittsburgh ou no questionário de sonolência de Epworth.

Fonte: Elaborada pelos autores (2021)

Dos 11 atletas avaliados, $8(72 \%)$ apresentam uma qualidade de sono ruim, ou seja, atingiram pontuações acima de 5 no questionário de Pittsburgh (PSQI). Apenas 3 voluntários (28\%) apresentaram uma boa qualidade de sono. Já o tempo total de sono (TTS) indicou que $6(54 \%)$ atletas dormem entre 7 a 9 horas por noite e $5(46 \%)$ atletas dormem menos de 7 horas. Com relação a eficiência do sono, identificou-se que 6 (54\%) destes atletas possuem um sono com eficiência abaixo de $85 \%$ e $5(46 \%)$ apresentaram eficiência superior a $85 \%$. No que se refere a latência do sono, 7 (63\%) atletas demoraram mais de 15 
minutos para o início do sono enquanto 4 (37\%) apresentaram uma latência menor que 15 minutos. E, apenas 3 (28\%) apresentaram sonolência diurna, ou seja, pontuação maior que 10 na escala de Epworth e 8 (72\%) dos atletas não apresentam sonolência diurna.

\section{Discussão}

O objetivo do presente estudo, é avaliar a qualidade do sono e o nível de sonolência de JBCR com lesão medular (LM). Os resultados demonstram que JBCR apresentam uma qualidade de sono ruim, porém sem sonolência diurna excessiva. Além disso, os JBCR apresentam um tempo total de sono (TTS) dentro dos valores preconizados pelo PSQI e os valores de latência do sono e eficiência do sono, muito próximos aos valores desejáveis para pessoas saudáveis sugerindo que a prática regular de exercício físico possa atenuar as alterações na qualidade e no padrão de sono em pessoas com LM.

Apesar dos resultados do estudo apontarem uma baixa qualidade do sono entre os JBCR, não podemos afirmar que estes atletas dormem mal, porque os valores atingidos no questionário de Pittsburgh foram muito próximos dos valores considerados satisfatórios para uma boa qualidade de sono.

Quanto a duração do sono, observou-se que os atletas dormem o tempo adequado, com mediana de 7,5 horas, confrontando com a literatura que afirma que indivíduos com LM tem uma duração de sono menor (Giannoccaro et al., 2013). Neste caso, podemos levantar a hipótese que, por se tratar de indivíduos fisicamente ativos, o exercício possa ter influenciado no sono destes atletas e consequentemente na duração. Entretanto, estudos afirmam que indivíduos treinados apresentam maior tempo total de sono do que pessoas sedentárias (Kelley \& Kelley, 2017; Martins et al., 2001) outros estudos reforçam que o sono de pessoas ativas é melhor do que o de pessoas sedentárias com a possibilidade de uma qualidade de sono melhor e menos cansaço durante o dia seguinte além da disposição para a prática de atividade física (Rombaldi \& Soares, 2016).

Embora a eficiência do sono tenha sido baixa, não podemos certificar que o sono dos JBCR seja ineficaz porque os valores obtidos estão muito próximos dos valores sugerido pelo questionário aplicado. Durante o sono o organismo recupera o desgaste físico e mental e, por isso um sono eficiente é fundamental para reparar estes desgastes gerados ao longo do dia (Fullagar et al., 2015). Estudos indicam que o exercício físico proporciona um sono melhor e restaurador fisicamente; e para Mello e Tufik (2004), o sono é considerado como um evento restaurador (Bonnar et al., 2018).

Os atletas que apresentaram baixa eficiência podem apresentar alguma particularidade específica referentes à LM, comprometendo o sono e sua função restauradora. Estudos afirmam que pessoas com LM apresentam alguns comprometimentos próprios da LM como, dor neuropática, depressão, complicações respiratórias e síndrome da apneia do sono (Alves et al., 2005). As causas mais comuns de prejuízo do sono são a restrição e fragmentação, sendo que a restrição do sono pode ser consequência do uso de medicamentos, condições pessoais e estilos de vida. A fragmentação, decorrente de um sono de quantidade e qualidade inadequadas, muitas vezes está relacionada a fatores médicos ou ambientais (Martins et al., 2001).

O estudo indicou que os JBCR demoram a dormir o que provavelmente deve estar associado a dores e complicações respiratórias. Corroborando com a literatura que afirma ao dizer que indivíduos com LM apresentam maior latência do sono. Porém o exercício físico contribui no aumento do tempo de sono de ondas lentas e diminuição da latência para o início do sono (Martins et al., 2001).

Foi observado, que os atletas não apresentaram sonolência diurna, confrontando com a literatura ao afirmar que indivíduos com traumatismo raquimedular apresentam maior sonolência durante o dia (Giannoccaro et al., 2013). Neste caso, podemos considerar a possibilidade de que o fator exercício físico tenha contribuído para diminuir a sonolência destes indivíduos, sendo que vários estudos apontam a influência significativa do exercício físico sobre o sono. Um levantamento epidemiológico realizado na cidade de São Paulo, demonstrou que entre 27,1 e 28,9\% de pessoas fisicamente ativas e 72,9 e 
$71,1 \%$ entre os sedentários se queixavam de insônia e sonolência excessiva, respectivamente, o que demonstra claramente a influência da atividade física na qualidade do sono (Martins et al., 2001; Santos-Silva et al., 2009).

Como resposta destes impactos, podemos apoiar na teoria da termorregulação e o aumento da dissipação de calor como elemento para promover a vigília (Antunes et al., 2008). Contudo, podemos afirmar que não houve sonolência porque os atletas dormem o tempo adequado em torno de 7 a 9 horas.

Entretanto, este estudo corrobora em alertar os treinadores e educadores físicos quanto aos benefícios do exercicio físico sobre o sono além do aumento da produção de melatonina, conhecido como o hormônnio do sono (Zisapel, 2018). Hábitos do sono adequados devem fazer parte da rotina de atletas em gera (Vitale et al., 2019). No entanto, sugere-se a recomendação, orientação e prescrição do exercício com essa finalidade em especial para indivíduos com LM.

Como limitação o estudo em questão apresentou algumas restrições específicas. Nesse caso, por se tratar de um estudo que avalia a qualidade subjetiva do sono, os resultados obtidos poderão ter erros que deverão ser considerados. A falta de um grupo controle de pessoas que não praticam atividade física sem LM, para comparar os resultados e fundamentar conclusões mais concretas. Nesse caso, seria interessante a utilização de métodos que mensuram de forma objetiva a qualidade do sono como a polissonografia, a actigrafia; e o teste múltiplo de latência do sono que permitiria estabelecer com maior clareza o padrão de sono e a sonolência.

\section{Conclusão}

Podemos concluir, que os JBCR apresentam uma qualidade de sono ruim, como demonstrado pelo PSQI. Entretanto nós acreditamos que a prática regular de exercício físico pode ter atenuado os efeitos deletérios da LM sobre o sono como demonstrado, citando como exemplo, os valores do TTS. Finalmente, a inclusão de um grupo controle ou a avaliação do sono por parâmetros objetivos e subjetivos, simultaneamente, poderia contribuir com um maior conhecimento em estudos envolvendo o sono em pessoas com lesão medular.

\section{Referências}

Alves, R., Veloso, S., Bizari, L., Carvalho, L., \& Fernandes, G. (2005). Qualidade do sono em indivíduos com traumatismo raquimedular. Revista Neurociências, 13. https://doi.org/10.4181/RNC.2005.13.178

Antunes, H. K. M., Andersen, M. L., Tufik, S., \& De Mello, M. T. (2008). Privação de sono e exercício físico. Revista Brasileira de Medicina do Esporte, 14, 51-56. https://doi.org/10.1590/S1517-86922008000100010

Antza, C., Kostopoulos, G., Mostafa, S., Nirantharakumar, K., \& Tahrani, A. (2021). The links between Sleep Duration, Obesity and Type 2 Diabetes Mellitus. The Journal of Endocrinology, JOE-21-0155.R1. https://doi.org/10.1530/JOE-21-0155

Bertolazi, A. N., Fagondes, S. C., Hoff, L. S., Dartora, E. G., Miozzo, I. C. da S., de Barba, M. E. F., \& Barreto, S. S. M. (2011). Validation of the Brazilian Portuguese version of the Pittsburgh Sleep Quality Index. Sleep Medicine, 12(1), 70-75. https://doi.org/10.1016/j.sleep.2010.04.020

Bertolazi, A. N., Fagondes, S. C., Hoff, L. S., Pedro, V. D., Menna Barreto, S. S., \& Johns, M. W. (2009). Validação da escala de sonolência de Epworth em português para uso no Brasil. Jornal Brasileiro de Pneumologia, 35, 877-883. https://doi.org/10.1590/S1806-37132009000900009

Bonnar, D., Bartel, K., Kakoschke, N., \& Lang, C. (2018). Sleep Interventions Designed to Improve Athletic Performance and Recovery: A Systematic Review of Current Approaches. Sports Medicine (Auckland, N.Z.), 48(3), 683-703. https://doi.org/10.1007/s40279-017-0832-x

Callegari-Jacques, S. M. (2003). Bioestatística: Princípios e Aplicações. Artmed.

Chennaoui, M., Arnal, P. J., Sauvet, F., \& Léger, D. (2015). Sleep and exercise: A reciprocal issue? Sleep Medicine Reviews, $20,59-72$. https://doi.org/10.1016/j.smrv.2014.06.008

Fullagar, H. H. K., Skorski, S., Duffield, R., Hammes, D., Coutts, A. J., \& Meyer, T. (2015). Sleep and athletic performance: The effects of sleep loss on exercise performance, and physiological and cognitive responses to exercise. Sports Medicine (Auckland, N.Z.), 45(2), 161-186. https://doi.org/10.1007/s40279-014-0260-0 
Research, Society and Development, v. 10, n. 16, e438101623761, 2021

(CC BY 4.0) | ISSN 2525-3409 | DOI: http://dx.doi.org/10.33448/rsd-v10i16.23761

Giannoccaro, M. P., Moghadam, K. K., Pizza, F., Boriani, S., Maraldi, N. M., Avoni, P., Morreale, A., Liguori, R., \& Plazzi, G. (2013). Sleep disorders in patients with spinal cord injury. Sleep Medicine Reviews, 17(6), 399-409. https://doi.org/10.1016/j.smrv.2012.12.005

Kelley, G. A., \& Kelley, K. S. (2017). Exercise and sleep: A systematic review of previous meta-analyses. Journal of Evidence-Based Medicine, 10(1), 26-36. https://doi.org/10.1111/jebm.12236

Martins, P. J. F., Mello, M. T. de, \& Tufik, S. (2001). Exercício e sono. Revista Brasileira de Medicina do Esporte, 7, 28-36. https://doi.org/10.1590/S151786922001000100006

Mello, M. T. de, \& Tufik, S. (2004). Atividade Física, Exercício Físico E Os Aspectos Psicobiológicos (1ª edição). Guanabara Koogan.

Müller, M. R., \& Guimarães, S. S. (2007). Impacto dos transtornos do sono sobre o funcionamento diário e a qualidade de vida. Estudos de Psicologia (Campinas), 24, 519-528. https://doi.org/10.1590/S0103-166X2007000400011

Nicolau, M. C., Akaârir, M., Gamundí, A., González, J., \& Rial, R. V. (2000). Why we sleep: The evolutionary pathway to the mammalian sleep. Progress in Neurobiology, 62(4), 379-406. https://doi.org/10.1016/s0301-0082(00)00013-7

Rombaldi, A. J., \& Soares, D. G. (2016). Indicadores da prática de atividade física e da qualidade do sono em escolares adolescentes. Revista Brasileira de Ciências do Esporte, 38(3), 290-296. https://doi.org/10.1016/j.rbce.2015.10.010

Sankari, A., Vaughan, S., Bascom, A., Martin, J. L., \& Badr, M. S. (2019). Sleep-Disordered Breathing and Spinal Cord Injury: A State-of-the-Art Review. Chest, 155(2), 438-445. https://doi.org/10.1016/j.chest.2018.10.002

Santos-Silva, R., Tufik, S., Conway, S. G., Taddei, J. A., \& Bittencourt, L. R. A. (2009). Sao Paulo Epidemiologic Sleep Study: Rationale, design, sampling, and procedures. Sleep Medicine, 10(6), 679-685. https://doi.org/10.1016/j.sleep.2008.11.001

Tobaldini, E., Costantino, G., Solbiati, M., Cogliati, C., Kara, T., Nobili, L., \& Montano, N. (2017). Sleep, sleep deprivation, autonomic nervous system and cardiovascular diseases. Neuroscience and Biobehavioral Reviews, 74(Pt B), 321-329. https://doi.org/10.1016/j.neubiorev.2016.07.004

Vitale, K. C., Owens, R., Hopkins, S. R., \& Malhotra, A. (2019). Sleep Hygiene for Optimizing Recovery in Athletes: Review and Recommendations. International Journal of Sports Medicine, 40(8), 535-543. https://doi.org/10.1055/a-0905-3103

Zisapel, N. (2018). New perspectives on the role of melatonin in human sleep, circadian rhythms and their regulation. British Journal of Pharmacology, 175(16), 3190-3199. https://doi.org/10.1111/bph.14116 